\title{
LEUKAEMIA
}

\section{Gene expression 1, histology 0}

Leukaemias of a single histological type acute lymphoblastic leukaemia (ALL), for instance - are a mixed bag when we consider their chromosomal translocations. But do all translocations have an effect on tumour behaviour, or are some an irrelevant consequence of chromosomal instability? Leukaemias with translocations that involve the mixedlineage leukaemia gene $(M L L)-\mathrm{a}$ transcription factor that is involved in haematopoietic cell development - are classified as ALL on the basis of histology, but Scott Armstrong and co-workers now present a strong case for redefining them as a new type of leukaemia.

The authors used oligonucleotide microarrays to compare gene-expression patterns in samples from 20 patients with conventional ALL versus 17 patients with the $M L L$ translocation. The two sets had distinct gene-expression patterns: the $M L L$ set underexpressed many genes involved in B-cell development, and overexpressed some genes from progenitor cells and myeloid cells. Might they be more closely related to acute myeloid leukaemia (AML) than to ALL? When the expression levels of 500 genes that best differentiate AML from ALL were used to classify the MLL samples, their expression pattern was distinct from those of both AML and ALL. This allowed the authors to define a predictor set of 100 genes that correctly classified 10 out of 10 new clinical samples.

By showing that a translocation can specify a gene-expression programme, Armstrong and colleagues have uncovered a new type of leukaemia that paves the way for the molecular classification of acute leukaemia. Patients with $M L L$ translocations respond poorly to standard therapy for ALL, but we now have a clear rationale for the development of new therapies aimed at treating acute leukaemias on the basis of their translocations, a strategy that has already proved successful for chronic myelogenous leukaemia.

Cath Brooksbank

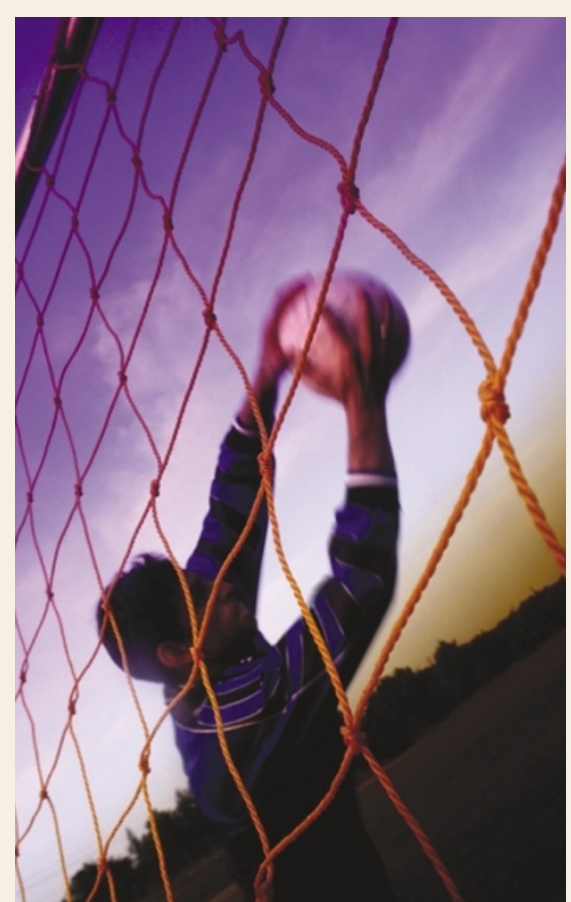

(2) References and links ORIGINAL RESEARCH PAPER Armstrong, S. A. et al. MLL translocations specify a distinct gene expression profile that distinguishes a unique leukaemia. Nature Genet. 30, 41-47 (2002)

\section{Unfold with BRCA1}

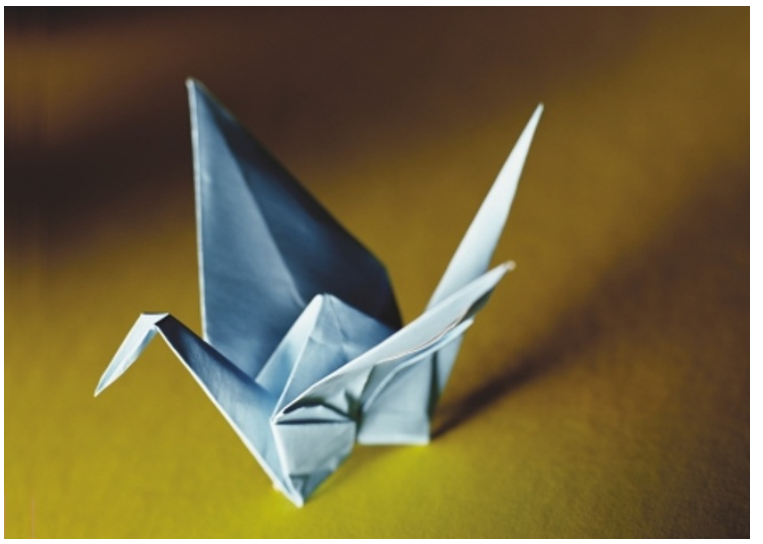

The multifunctional nature of BRCA1 has led many to believe that it regulates processes such as transcription and DNA repair by a common mechanism. Now, Rong Li and colleagues, reporting in the 10 December issue of The Journal of Cell Biology, provide evidence indicating that this mechanism is chromatin unfolding, and that it is altered by many cancer-predisposing BRCA1 mutations.
To investigate the effect of BRCA1 on chromatin structure, the authors used a cell line containing many copies of the lac operator, which forms a large heterochromatic region. Immunofluoresence using antibodies against the lac repressor - which binds to the lac operator sequences - allows the region to be visualized as a compact nuclear dot. Expression of a BRCA1-lac repressor fusion protein alters this structure in $14 \%$ of cells: it becomes irregularly shaped, indicative of chromatin unfolding. The authors used deletion analysis to identify three regions of BRCA1 that could induce this unfolding in BRCAl's carboxy-terminal transcriptional activation domain. They include activation domain 1 (AD1) and the two BRCA1 C-terminal repeats (BRCT1 and BRCT2), which lie within $\mathrm{AD} 2$.

Histone modification is a known chromatin-regulation mechanism, so is this involved in BRCA1-mediated regulation? Unlike p53 and E2F1 - two other transcription factors that induce chromatin unfolding - BRCA1 does not induce hyperacetylation of histones $\mathrm{H} 3$ and $\mathrm{H} 4$. It does, however, induce phosphorylation of $\mathrm{H} 2 \mathrm{AX}$, a variant of histone $\mathrm{H} 2 \mathrm{~A}$, although this has not been directly linked to chromatin regulation.

So how is this chromatin regulation related to cancer-predisposing BRCA1 mutations? When classified according to their effect on chromatin unfolding, BRCA1 mutations fall into three classes, two of which have an effect on chromatin regulation. Nonsense mutations result in C-terminal truncations that are defective in transcription and repair, and do not show chromatin unfolding, and mutations within AD2 result in an enlargement of the unfolded chromatin structure and an increase in the number of cells with unfolded chromatin.

What remains to be determined is whether the BRCA1 mutation classes that cause different effects on chromatin unfolding also lead to different effects on risk, types or prognosis of BRCA1-associated cancers.

Emma Greenwood

\section{(2) References and links}

ORIGINAL RESEARCH PAPER Ye, Q. et al. BRCA1-induced large-scale chromatin unfolding and allele-specific effects of cancer-predisposing mutations. J. Cell Biol. 155, 911-921

(2001)

WEB SITE

Rong Li's lab: http://hsc.virginia.edu/bmg/faculty/li/li.html 